\title{
Modeling of Circuits within Networks by fMRI
}

\author{
G. de Marco, A. le Pellec \\ Université Paris $X$, Laboratoire du contrôle moteur et d'analyse du mouvement, Paris, France \\ E-mail:demarco.giovanni@gmail.com \\ Received November 24, 2009; revised December 19, 2009; accepted January 8, 2010
}

\begin{abstract}
In this review, the authors describe the most recent functional imaging approaches used to explore and identify circuits within networks and model spatially and anatomically interconnected regions. After defining the concept of functional and effective connectivity, the authors describe various methods of identification and modeling of circuits within networks. The description of specific circuits in networks should allow a more realistic definition of dynamic functioning of the central nervous system which underlies various brain functions.
\end{abstract}

Keywords: fMRI, CNS, Modeling, Network, Effective Connectivity

\section{Introduction}

Imaging can be used to locate the brain areas involved in various forms of motor behavior, attention, vision or emotion, self-awareness and awareness of others, but brain network modeling probably remains the greatest challenge in the field of imaging data analysis [1]. Neuroimaging first allowed researchers to describe the cortical and subcortical activity of regionally segregated functional regions during a variety of experimental or cognitive tasks. More recently, functional integration studies have described how these functionally specialized areas, i.e., areas whose activity is temporally modified, interact within a highly distributed neural network. By using functional magnetic resonance imaging (fMRI), which has become the method most commonly used to investigate human brain functions and define neural populations as distributed local networks transiently, linked by large-scale reciprocal dynamic connections [2].

After defining the concept of functional and effective connectivity, various approaches to the identification and modeling of circuits into networks will be presented in order to more realistically define the dynamics of the central nervous system which underlies various cerebral functions. A distinction should be made between methods that only consider correlations and ignore issues of causality and influence and methods that attempt to describe or draw inferences concerning the direction of influence between regions. Methodological approaches to the study of connectivity using fMRI data may be broadly divided into those that are more data-driven and attempt to map connectivity in the whole brain and those that use prior knowledge or hypotheses-driven, limited to a restricted set of regions [3]. These two categories of analysis are described, as indicated below, as functional connectivity and effective connectivity, respectively [4-6]. Techniques in the first group that consider only correlations between regions include mapping using seed-voxel correlations. Techniques in the second group use more elaborate models and additional assumptions applied to calculate correlations or covariances to address questions about directional influences and include mapping based on structural equation modeling (SEM), multivariate autoregressive (MAR) modeling, dynamic causal modeling (DCM).

\section{Functional and Effective Connectivity}

The dichotomy between local and large-scale networks serves as a neural basis for the key assumption that brain functional architecture abides by two principles: functional segregation and functional integration [2,3,7]. A large-scale brain network can be defined as a set of segregated and integrated regions that share strong anatomical connections and functional interactions. Whether top-down or bottom-up, connections and interactions are quintessential aspects of networks [8,9]. Cognitive and sensorimotor processes depend on complex dynamics of temporally and spatially segregated brain activities. While the segregation principle states that some functional processes specifically engage well-localized and specialized brain regions, it is now thought that brain functions are most likely to emerge through integration of information flows across widely distributed regions $[2,10,11]$. According to this approach, it is not only isolated brain areas that are presumed to process informa- 
tion but rather a large-scale network, i.e. a set of brain regions interacting in a coherent and dynamic way. Hence, according to the functional integration concept, cortical areas and therefore functions are integrated within specific dynamic networks.

This concept supposes the existence of a dynamic interaction between interconnected, active areas and that the brain areas are expressed as networks within integrated systems. In such a system, localized areas are included in networks which become dynamic according to the cognitive task. Brain areas underlie several functions and can belong successively to several different functional networks. In other words, a given brain area does not have a single function; its resources can be exploited in several different cognitive strategies. The principle of functional integration which is also known in the field of electrophysiology was used to analyze the event potentials obtained from multielectrode recordings [12]. Thus, based on the functional integration principle, the relationships between several brain areas may be examined.

Effective connectivity, closer to the intuitive notion of a connection, can be defined as the influence that one neural system exerts over another, either at a synaptic level (synaptic efficacy) or a cortical level $[13,14]$. This approach emphasizes that determining effective connectivity requires a causal model of the interactions between the elements of the neural system of interest. In electrophysiology, there is a close relationship between effective connectivity and synaptic efficacy [15]. Effective connectivity can be estimated from linear models to test whether a theoretical model seeking to explain a network of relationships can actually fit the relationships estimated from the observed data. In the case of fMRI, the theoretical model is an anatomically constrained model and the data are interregional covariances of activity [16].

Consequently, effective connectivity represents the dynamic influence that cortical and subcortical regions exert on each other via a putative network of interdependent areas $[5,12]$. This approach might be based on linear time-invariant models that relate the time-course of experimentally controlled manipulations to BOLD signals in a voxel-specific fashion. Although various statistical models have been proposed [17], these standard models treat the voxels throughout the brain as isolated black boxes, whose input-output functions are characterized by BOLD responses evoked by various experimental conditions [18]. fMRI provides simultaneous recordings of activity throughout the brain evoked by cognitive and sensorimotor challenges, but at the expense of ignoring temporal information, i.e., the history of the experimental task (input) or physiologic variable (signal). This is important, as interactions within the brain, whether over short or long distances, take time and are not instantaneous which is implicit within regression models. Furthermore, the instantaneous state of any brain system that conforms to a dynamic system will depend on the history of its input.

\section{Data-Driven Approaches}

The first category of methods includes seed-voxel correlations, Granger causality derived autoregressive models [19], fuzzy clustering which assumes that brain voxels can be grouped into clusters sharing similar activity patterns [20-22], hierarchical clustering [23,24], psychophysiologic interactions which test for changes in the regression slope of activity at every voxel on a seed voxel that are induced by an experimental manipulation [25], and spectral analysis [26-28]. Other techniques, such as principal component analysis [29-31] and independent component analysis (ICA) [32-35], suppose that fMRI data are a linear mixing of a given number of temporal factors with an associated factor-specific spatial distribution. Among all of these methods, we propose to briefly describe the ICA method (time analysis of the BOLD response) and the spectral method (frequency analysis of the time response) that are two interesting methods to spatially identify circuits within networks in the brain.

\subsection{Independent Component Analysis}

Independent component analysis (ICA) is a data-based multivariate statistical technique that uses higher order statistics to perform decomposition of linearly combined statistically independent sources [36]. Each statistically independent component represents a hemodynamic map of the whole brain. Each independent component is supposed to describe a particular functional activity of the brain with its deployment over time [37-39]. Each independent component extracted by applying a spatial ICA is spatially independent of all other independent components [35]. Therefore, the contribution of a spatial independent component to each voxel is given by the independent component magnitude at that point modulated over time by the associated time-course. The main advantage of ICA is that it requires little knowledge about the nature of the data. The only necessary hypothesis concerns the presence of a sufficient amount of independent sources (temporal or spatial), which are linearly mixed. Conversely, one of the main drawbacks of ICA is the large amount of brain activations resulting from this kind of decomposition [40]. At some point, hypotheses are necessary to select relevant from spurious activations.

For this reason, ICA can be used in conjunction with other well-established techniques [41] or further information may be associated with the reference time-course, such as the spatial localization of activities [42] and the covariate relation of independent component time-course 
[43]. ICA could be combined with SEM to extend the explanatory power of each technique. SEM is a well developed, computationally minimally intensive connectivity analysis technique suitable for neuroimaging data, especially when it is combined with other data-driven methods such as ICA. In this case, SEM coupled with ICA is capable to handle data from a large number of subjects [32]. The biological relevance and cortical connections of the SEM models have also been evaluated with reference to available knowledge based on animal and human circuitries. The main advantage of spatial group ICA is its ability to identify the distinct functional elements involved in the circuitry [33]. Functionally connected brain regions encompassed in each independent component are active at the same time, suggesting that one or more anatomical connections are in use during performance of the task. Although this reasoning is more in line with the "connectionist" approach to brain functions based on parallel processing mechanisms performed by a group of connected functional elements, the ICA approach lacks a statistical method to model the functional connections assumed to exist between regions. The addition of ICA to SEM can address this issue. Each ICA map or part of the map corresponds to one component in an SEM.

\subsection{Spectral Analysis}

The description of a correlation structure in the frequency domain can be a promising approach to investigate interregional strengths of interactions of a functional network. As time-dependent correlations may vary between fMRI signals and across the space independently of the underlying neural dynamics, a method of analysis of frequency-dependent correlations would be one way to overcome this interregional variability of the BOLD response and would also be crucial for extracting the fine detail of information hidden within the fMRI signal. Functional connectivity analysis in the presence of major physiologic noise sources is a pitfall especially when the correlation (or covariance) between BOLD signals is performed in the time domain. In this case, these noise sources may artificially increase the magnitude of cross-correlation. Estimation of coherence between pairs of voxels at a specific frequency or at a limited range of frequencies can therefore be one way to deal with noxious physiologic noise.

The frequency domain approach can be used to analyze a limited range of linear relationships within a restricted frequency band [44]. Consequently, measurement of the correlation between fMRI data can be enhanced and can help to resolve the problem of false connectivity derived from cardiac and respiratory cycles and/or vascular differences. This approach can be performed by using spectral analysis, which allows exami- nation of the structure of covariance and provides certain voxel-based parameters such as coherence which assesses the dependence between voxel signals [26].

The spectral theory for multivariate time series has already been used in several fMRI studies $[27,28,45]$. By using fMRI signals, these authors demonstrated that time domain approaches may be sufficiently susceptible to substantially high frequency artefacts, whereas the spectral domain is essentially resistant to these artefacts. They also demonstrated that the frequency-dependent correlation is higher than that measured in the temporal domain. In other fields of neuroscience, for instance in electroencephalography (EEG), coherence analysis is widely used to investigate correlated oscillatory activities between various areas in the brain [46-49]. In magnetoencephalography (MEG), coherence analysis has also been demonstrated to be a useful technique in clinical studies for discriminating different rhythmic behaviors in various brain regions [50-52]. However, although the relationship between neuronal currents and hemodynamic response is poorly understood, simultaneous intracortical neural recordings and fMRI signals acquired in animals recently revealed a significant correlation between local field potential and vascular response [53]. The feasibility of a correlation between the synchrony of low frequency BOLD fluctuations in functionally related brain regions and neuronal connections that facilitate coordinated activities has been demonstrated in various applications $[54,55]$.

\section{Hypothesis-Driven Approaches}

The alternative to data-based approaches is to use a model that attempts to describe the relationships between a set of selected regions, in which region-specific measurements such as BOLD time series are extracted from whole-brain data prior to the connectivity modeling stage. This category includes structural equation modeling (SEM) [56-62], multivariate autoregressive (MAR) modeling [63,64], dynamic causal modeling (DCM) [65-67], generative models including neural mass models $[68,69]$ and large-scale neural models [70-72].

\subsection{Structural Equation Modelling}

Path analysis, also referred to as structural equation modeling (SEM), was originally developed in the early 1970s by Jöreskog, Keesling, and Wiley, when they combined factor analysis with econometric simultaneous equation models [73-76]. In the early 1990s, McIntosh introduced SEM to neuroimaging [56,59,77-79] for modeling, testing, and comparison of directional effective connectivity of the brain. SEM rapidly became popular in this field [31,57,80-86]. Structural models can be used to analyze linear relationships between variables 
from analysis of the covariance among the variables. Structural models were developed from two principal methods of analyses: factorial analysis (for a review: [75]) and multiple regression or causal path analysis (a method developed in the 1930s by Wright e.g., (for a review: [87]). Structural models examine multiple sources of influence on the dependent variable in an experiment $[88,89]$.

Structural Equation Modeling (SEM) is a hypothesis-based multivariate statistical technique of data analysis that can be used with neuroimaging data. An increasing number of PET, fMRI and transcranial magnetic stimulation (TMS) studies have used SEM to investigate large-scale functional brain networks [90-93] and show specific networks involved in either working memory [94-100], attentional processes [64,101-103], face perception [104-106], motor movement processing [61, $107-112]$, language $[32,113,114]$ or processing of painful stimuli [62].

SEM methods, in comparison with classical approaches such as linear regression, allow simultaneous analysis of several types of interrelationships between variables in an experiment [13,115-117]. The nature of the relationship between variables is given by the regression coefficient; it describes how much the dependent variable changes when an independent variable changes by one unit. SEM directly integrates measurement errors into a statistical model, so that estimates of regression coefficients are consequently more precise than with classical methods such as multiple regression, factorial analysis, or analysis of variance. The older methods examine only one linear relationship at the same time between independent and dependant variables and only within a range of values set by the investigator [14]. In contrast with classical methods, SEM analyzes a structure of variances and covariances in a dataset of observed variables and can be used to predict dependences between variables. In other words, SEM seeks to explain as much of the variance in dependant variables as it can from simultaneous measurement of the variances of the independent variables included in the model. Similarly, SEM incorporates measurement errors of the independent variables into calculation of the estimate, which reinforces the statistical power of the method and provides more precise estimates of regression coefficients. A model of measurement can therefore be validated from a theoretical model or empirical data [99]. The objective of effective connectivity analysis is to estimate parameters that represent influences between regions that may change over time and with respect to experimental tasks.

In order to describe a functional network, network nodes and anatomical connections must therefore be proposed in conjunction with a SEM model to explain interregional covariances and determine the intensity of the connections. When applied to PET or fMRI data, SEM allows modeling of connection pathways between cortical or subcortical areas and reveals relationships, interdependencies and covariance between the various areas. In a given anatomical model, SEM shows the effects of an experimental task on a specific network of connections [14,118-120]. In this type of statistical analysis, normalized variables are considered in terms of the structure of their covariances. SEM therefore allows inference of interregional dependencies between various cerebral cortical areas.

SEM is a simple and pragmatic approach to effective connectivity when dynamic aspects can be disregarded. A linear model is sufficient and the observed variables can be measured precisely, the input is unknown but stochastic and stationary. SEM comprises a set of regions and a set of directed connections. Importantly, a causal relationship is ascribed to these connections. Causal relationships are therefore not inferred from the data, but are assumed a priori. The strengths of connections can therefore be set so as to minimize the discrepancy between observed and implied correlations and thereby fit a model to the data. Changes in connectivity can be attributed to experimental manipulation by partitioning the data set. If, for example, a given fMRI data set is partitioned into those scans obtained for different levels of an experimental factor, differences in connectivity can then be attributed to that factor leading to the conclusion that a pathway has been activated. An SEM with particular connection strengths implies a particular set of instantaneous correlations between regions. Structural equation models posit a set of theoretical causal relationships between variables and model instantaneous correlations i.e., correlations between regions at the same time-point. Instantaneous activity is assumed to be the result of local dynamics and connections between regions.

\subsection{Multivariate Autoregressive (MAR) Models}

To overcome the difficulties of SEM, Harrison et al. proposed the use of multivariate autoregressive (MAR) models for the analysis of fMRI data [63]. They were the first to introduce multivariate autoregressive (MAR) models into brain pathway analyses to characterize interregional dependence. MAR models are time-series models and consequently model temporal order within measured brain activity. Goebel et al. [19] and Roebroeck et al. [121] subsequently generalized the MAR approach by incorporating Granger causality between two time series. MAR models posit a set of causal relationships between variables; they incorporate cross-covariances between regions (covariances at multiple lags) and exploit temporal relationships between different scans to allow conclusions about predominant directions of influence between regions as well as their strength [18, 122,123]. 
An autoregressive approach is used to characterize a structure in a time series, whereby the current value of a time series is modeled as a weighted linear sum of previous values. Consecutive measurements within a given time series contain information about the process that generated this series. This is an autoregressive process and is a very simple, yet effective, approach to time series characterization. This is distinct from regression techniques that quantify instantaneous correlations, but is similar to the SEM model in that it estimates the relative influences over time. Autoregressive models of fMRI data address the temporal aspect of causality in a BOLD time series, focusing on the causal dependence of the present on the past. Each data point of a time series is explained as a linear combination of past data points. This approach contrasts with SEM regression-based models in which the time series can be permuted without changing the results. MAR models contain directed influences among a set of regions whose causal interactions, expressed at the BOLD level, are inferred via their mutual predictability from past time points.

\subsection{Dynamic Causal Modeling}

A major criticism of SEM or MAR with regard to neuroimaging data is that they model effective connectivity changes at the "hemodynamic level" rather than the "neuronal level". This is a serious problem because the causal architecture of the system that we want to identify is expressed in terms of neuronal dynamics, which are not directly observed using noninvasive techniques. In the case of fMRI data, previous models of effective connectivity have been fitted to the measured time series which result from a hemodynamic convolution of the underlying neural activity. Since classical statistical models do not include the forward model linking neuronal activity to the measured hemodynamic data, analyses of interregional connectivity performed on hemodynamic responses are problematic. For example, different brain regions can exhibit marked differences in neurovascular coupling, and these differences, expressed in different latencies (see above) may lead to false inferences about connectivity [124].

Dynamical Causal Modeling (DCM) has recently been developed as a generalization of both convolution models and SEM [66,67]. As described in Penny et al. [66], SEM can be shown to be a simplified version of DCM which also depends on the definition of a structural model. DCM model assumes a dynamic neuronal model of interacting brain regions, whereby neuronal activity in a given brain region causes changes in neuronal activity in other regions according to the structural model. This neuronal model is then supplemented with a forward model of how neuronal activity generates a measured BOLD response through the balloon model which was initially formulated by Buxton et al. [125] and later extended by Friston et al. [126]. A Bayesian inference scheme is devised to infer the model parameters from the data. The mathematical framework of DCM takes into account nonlinearities and temporal correlations. It also quantifies the interaction strength that one brain region exerts on another brain region at the neuronal level, whereas SEM only concerns the observed BOLD signal. DCM is suspected to be less sensitive than SEM to the number of degrees of freedom. Unlike SEM, DCM also models the effect of experimental, external, and modulatory inputs on network dynamics. Since DCM models neurobiologically plausible neural activities and takes into account dynamics and modulations, this mathematical framework would appear to be more advantageous than SEM.

\subsection{Diffusion Tensor Imaging}

While fMRI provides detailed information about the spatial location of functionally active cortical areas, the question of anatomical interdependency between cortical areas remains elusive. A key tool to assess the validity of large-scale distributed networks in fMRI is knowledge of the underlying anatomical connections. The original idea behind SEM and functional neuroimaging was to combine two data sets: a functional set with an anatomical set (connections between regions), based on the assumption that anatomy was the source of spatial causal relationships. Our understanding of the connections between regions is limited, but since the advent of newer tractography methods, the main white matter tracts can be described. Diffusion Tensor Imaging (DTI) is a powerful MRI technique $[127,128]$ that can be used to translate self-diffusion, or microscopic motion of water molecules in tissue into a MRI measure of tissue integrity and structure (white matter fibers). Data from diffusion tensor imaging (DTI) and fMRI have been combined in a few previous studies [129-131]. These studies showed that a combination of techniques can give additional information about brain organization which may give more specific information about organization of brain functions and brain injuries. In this latter case, a DTI-driven SEM would integrate information about white matter changes (e.g. maturation, aging) [100,132]. The prospect of using information derived from tractography could be used to constrain structural models. DTI and fMRI combinations will be essential to discover to what extent the brain functional organization as investigated with fMRI reflects structural features of the brain and, hence, to more accurately assess the relevance of fMRI to examine the relationship between functional and large-scale anatomical networks. However, more studies are still needed to investigate anatomical correlates which would be related to effective connectivity. 


\section{Conclusions}

This article describes the most recent imaging approaches used to explore and identify circuits within networks and to spatially and anatomically model interconnected regions. Structural equation modeling is the most widely used method to model effective connectivity $[56,82,133]$. The relevance of applying SEM to fMRI neuroimaging data has been discussed in detail elsewhere $[58,66,82,134]$. SEM allows one to start with simpler models and then progress to more complex models by repeatedly testing the model fit to real data. SEM is useful when some information is available, such as a small set of potential structural models or partial information concerning connectivity. Newer, more sophisticated effective connectivity analysis methods such as Dynamic Causal Modeling might circumvent the drawbacks of SEM and may shed more insight into how brain regions interact in information processing. Nevertheless, SEM is a well developed, computationally less intensive connectivity analysis technique suitable for neuroimaging data especially for block designs and combined with other methods such as independent component analysis, partial correlation or DTI. The use of SEM may be justified by the fact that, unlike DCM, the statistical model underlying SEM is quite simple and not computationally demanding.

\section{References}

[1] K. E. Stephan, et al., "Models of functional neiroimaging data," Center for Molecular Imaging Research, Vol. 2, pp. 15-34, 2006.

[2] F. Varela, et al., "The brainweb: Phase synchronization and large-scale integration," Nature Reviews Neuroscience, Vol. 2, No. 4, pp. 229-239, 2001.

[3] G. Marrelec, P. Bellec, and H. Benali, "Exploring largescale brain networks in functional MRI," Journal of Physiology Paris, Vol. 100, No. 4, pp. 171-181, 2006.

[4] B. Horwitz, et al., "Investigating the neural basis for functional and effective connectivity. Application to fMRI," Philosophical Transactions of the Royal Society B: Biological Sciences, Vol. 360, No. 1457, pp. 10931108, 2005.

[5] K. J. Friston, C. D. Frith, and R. S. J. Frackowiak, "Time-dependent changes in effective connectivity measured with PET," Human Brain Mapping, Vol. 1, pp. 69-79, 1993.

[6] A. M. Kelly and H. Garavan, "Human functional neuroimaging of brain changes associated with practice," Cerebral Cortex, Vol. 15, No. 8, pp. 1089-1102, 2005.

[7] B. Horwitz, M. A. Tagamets, and A. R. McIntosh, "Neural modeling, functional brain imaging, and cognition," Trends in Cognitive Sciences, Vol. 3, No. 3, pp. 91-98, 1999.
[8] M. M. Mesulam, "From sensation to cognition," Brain, Vol. 121, No. Pt 6, pp. 1013-1052, 1998.

[9] S. L. Bressler, "Large-scale cortical networks and cognition," Brain Research Reviews, Vol. 20, No. 3, pp. 288304, 1995.

[10] G. Tononi, G. M. Edelman, and O. Sporns, "Complexity and coherence: Integrating information in the brain," Trends in Cognitive Sciences, Vol. 2, pp. 474-484, 1998.

[11] O. Sporns, et al., "Organization, development and function of complex brain networks," Trends in Cognitive Science, Vol. 8, No. 9, pp. 418-425, 2004.

[12] G. L. Gerstein and D. H. Perkel, "Simultaneously recorded trains of action potentials: Analysis and functional interpretation," Science, Vol. 164, No. 881, pp. 828-830, 1969.

[13] K. Friston, "Functional and effective connectivity in neuroimaging: A synthesis," Human Brain Mapping, Vol. 2, pp. 56-78, 1994.

[14] A. McIntosh and F. Gonzalez-Lima, "Structural equation modeling and its application to network analysis in functional brain imaging," Human Brain Mapping, Vol. 2, pp. 2-22, 1994.

[15] A. Aersten and H. Preissl, "Dynamics of activity and connectivity in physiological neuronal networks," In: $\mathrm{H}$. G. Schuster, Ed., NonLinear Dynamics and Neuronal Networks, VCH Publishers Inc., New York, pp. 281-302, 1991.

[16] C. Buchel and K. Friston, "Assessing interactions among neuronal systems using functional neuroimaging," Neural Networks, Vol. 13, No. 8-9, pp. 871-82, 2000.

[17] R. Henson, "Analysis of fMRI time series: Linear timeinvariant models, event-related fMRI, and optimal experimental design," In: R. Frackowiack et al. Ed., Human Brain Function, 2nd edition, Elsevier, San Diego, pp. 793-823, 2004.

[18] K. E. Stephan, et al., "Biophysical models of fMRI responses," Current Opinion Neurobiology, Vol. 14, No. 5, pp. 629-635, 2004.

[19] R. Goebel, et al., "Investigating directed cortical interactions in time-resolved fMRI data using vector autoregressive modeling and Granger causality mapping," Magnetic Resonance Imaging, Vol. 21, No. 10, pp. 1251-1261, 2003.

[20] R. Baumgartner, et al., "Comparison of two exploratory data analysis methods for fMRI: Fuzzy clustering vs. principal component analysis," Magnetic Resonance Imaging, Vol. 18, No. 1, pp. 89-94, 2000.

[21] R. Baumgartner, et al., "Resampling as a cluster validation technique in fMRI," Journal of Magnetic Resonance Imaging, Vol. 11, No. 2, pp. 228-231, 2000.

[22] R. Baumgartner, C. Windischberger, and E. Moser, "Quantification in functional magnetic resonance imaging: Fuzzy clustering vs. correlation analysis," Magnetic Resonance Imaging, Vol. 16, No. 2, pp. 115-125, 1998.

[23] D. Cordes, et al., "Hierarchical clustering to measure connectivity in fMRI resting-state data," Magnetic Resonance Imaging, Vol. 20, No. 4, pp. 305-317, 2002. 
[24] C. Goutte, et al., "On clustering fMRI time series," Neuroimage, Vol. 9, No. 3, pp. 298-310, 1999.

[25] K. J. Friston, et al., "Psychophysiological and modulatory interactions in neuroimaging," Neuroimage, Vol. 6, No. 3, pp. 218-229, 1997.

[26] S. Fall and G. de Marco, "Assessment of brain interactivity in the motor cortex from the concept of functional connectivity and spectral analysis of fMRI data," Biological Cybernetics, Vol. 98, No. 2, pp. 101-114, 2008.

[27] F. T. Sun, L. M. Miller, and M. D'Esposito, "Measuring temporal dynamics of functional networks using phase spectrum of fMRI data," Neuroimage, Vol. 28, No. 1, pp. 227-237, 2005.

[28] K. Muller, et al., "On multivariate spectral analysis of fMRI time series," Neuroimage, Vol. 14, No. 2, pp. 347-356, 2001.

[29] K. J. Friston, et al., "Functional connectivity: The principal-component analysis of large (PET) data sets," Journal of Cerebral Blood Flow \& Metabolism, Vol. 13, No. 1, pp. 5-14, 1993.

[30] A. H. Andersen, D. M. Gash, and M. J. Avison, "Principal component analysis of the dynamic response measured by fMRI: A generalized linear systems framework," Magnetic Resonance Imaging, Vol. 17, No. 6, pp. 795815,1999

[31] E. T. Bullmore, et al., "Functional magnetic resonance image analysis of a large-scale neurocognitive network," Neuroimage, Vol. 4, No. 1, pp. 16-33, 1996.

[32] P. R. Karunanayaka, et al., "Age-related connectivity changes in fMRI data from children listening to stories," Neuroimage, Vol. 34, No. 1, pp. 349-360, 2007.

[33] N. Correa, T. Adali, and V. D. Calhoun, "Performance of blind source separation algorithms for fMRI analysis using a group ICA method," Magnetic Resonance Imaging, Vol. 25, No. 5, pp. 684-694, 2007.

[34] V. D. Calhoun, et al., "Latency (in) sensitive ICA. Group independent component analysis of fMRI data in the temporal frequency domain," Neuroimage, Vol. 20, No. 3, pp. 1661-1669, 2003.

[35] M. J. Jafri, et al., "A method for functional network connectivity among spatially independent resting-state components in schizophrenia," Neuroimage, Vol. 39, No. 4, pp. 1666-1681, 2008.

[36] A. Hyvärinen, "Fast and robust fixed-point algorithms for independent component analysis," IEEE Transactions on Neural Networks, Vol. 10, pp. 626-634, 1999.

[37] F. Esposito, et al., "Spatial independent component analysis of functional MRI time-series: To what extent do results depend on the algorithm used?" Human Brain Mapping, Vol. 16, No. 3, pp. 146-157, 2002.

[38] C. F. Beckmann and S. M. Smith, "Probabilistic independent component analysis for functional magnetic resonance imaging," IEEE Transactions on Medical Imaging, Vol. 23, No. 2, pp. 137-152, 2004.

[39] M. J. McKeown and T. J. Sejnowski, "Independent com- ponent analysis of fMRI data: Examining the assumptions," Human Brain Mapping, Vol. 6, No. 5-6, pp. 368-372, 1998.

[40] M. J. McKeown, et al., "Analysis of fMRI data by blind separation into independent spatial components," Human Brain Mapping, Vol. 6, No. 3, pp. 160-188, 1998.

[41] D. Hu, et al., "Unified SPM-ICA for fMRI analysis," Neuroimage, Vol. 25, No. 3, pp. 746-755, 2005.

[42] B. Hong, G. D. Pearlson, and V. D. Calhoun, "Source density-driven independent component analysis approach for fMRI data," Human Brain Mapping, Vol. 25, No. 3, pp. 297-307, 2005.

[43] M. J. McKeown, "Detection of consistently task-related activations in fMRI data with hybrid independent component analysis," Neuroimage, Vol. 11, No. 1, pp. 24-35, 2000

[44] F. T. Sun, L. M. Miller, and M. D'Esposito, "Measuring interregional functional connectivity using coherence and partial coherence analyses of fMRI data," Neuroimage, Vol. 21, No. 2, pp. 647-458, 2004.

[45] L. J. Marchini and B. D. Ripley, "A new statistical approach to detecting significant activation in funtional MRI," Neuroimage, Vol. 12, pp. 366-380, 2000.

[46] C. Andrew and G. Pfurtscheller, "Event-related coherence as a tool for studying dynamic interaction of brain regions," Electroencephalography and Clinical Neurophysiology, Vol. 98, No. 2, pp. 144-148, 1996.

[47] J. Classen, et al., "Integrative visuomotor behavior is associated with interregionally coherent oscillations in the human brain," Journal of Neurophysiology, Vol. 79, No. 3, pp. 1567-1573, 1998

[48] F. G. Andres, et al., "Functional coupling of human cortical sensorimotor areas during bimanual skill acquisition,” Brain, Vol. 122, No. Pt 5, pp. 855-870, 1999.

[49] P. Rappelsberger and H. Petsche, "Probability mapping: Power and coherence analyses of cognitive processes," Brain Topography, Vol. 1, No. 1, pp. 46-54, 1988.

[50] T. Locatelli, et al., "EEG coherence in Alzheimer's disease," Electroencephalography and Clinical Neurophysiology, Vol. 106, No. 3, pp. 229-237, 1998.

[51] K. Le Roc'h, "EEG coherence in Alzheimer disease, by Besthorn et al.," Electroencephalography and Clinical Neurophysiology, Vol. 91, No. 3, pp. 232-233, 1994.

[52] C. Besthorn, et al., "EEG coherence in Alzheimer disease," Electroencephalography and Clinical Neurophysiology, Vol. 90, No. 3, pp. 242-245, 1994.

[53] N. K. Logothetis, et al., "Neurophysiological investigation of the basis of the fMRI signal," Nature, Vol. 412, No. 6843, pp. 150-157, 2001.

[54] V. Haughton and B. Biswal, "Clinical application of basal regional cerebral blood flow fluctuation measurements by fMRI," Advances in Experimental Medicine and Biology, Vol. 454, pp. 583-590, 1998.

[55] D. Cordes, et al., "Mapping functionally related regions of brain with functional connectivity MR imaging," American Journal of Neuroradiology, Vol. 21, No. 9, pp. 
1636-1644, 2000.

[56] A. R. McIntosh and F. Gonzalez-Lima, "Structural equation modeling and its application to network analysis in functional brain imaging," Human Brain Mapping, Vol. 2, pp. 2-22, 1994.

[57] M. Glabus, et al., "Interindividual differences in functional interactions among prefrontal, parietal and parahippocampal regions during working memory," Cerebral Cortex, Vol. 13, pp. 1352-1361, 2003.

[58] M. S. Goncalves and D. A. Hall, "Connectivity analysis with structural equation modelling: An example of the effects of voxel selection," Neuroimage, Vol. 20, No. 3, pp. 1455-1467, 2003.

[59] A. McIntosh, et al., "Network analysis of cortical visual pathways mapped with PET," Journal of Neuroscience, Vol. 14, pp. 655-666, 1994.

[60] C. Büchel and K. Friston, "Assessing interactions among neuronal systems using functional neuroimaging," Neural Networks, Vol. 13, No. 8-9, pp. 871-882, 2000.

[61] T. Taniwaki, et al., "Age-related alterations of the functional interactions within the basal ganglia and cerebellar motor loops in vivo," Neuroimage, Vol. 36, No. 4, pp. 1263-1276, 2007.

[62] J. G. Craggs, et al., "Functional brain interactions that serve cognitive-affective processing during pain and placebo analgesia," Neuroimage, Vol. 38, No. 4, pp. 720 729, 2007.

[63] L. Harrison, D. Penny, and K. Friston, "Multivariate autoregressive modeling of fMRI time series," Neuroimage, Vol. 19, No. 4, pp. 1477-1491, 2003.

[64] J. Kim, et al., "Unified structural equation modeling approach for the analysis of multisubject, multivariate functional MRI data," Human Brain Mapping, Vol. 28, No. 2, pp. 85-93, 2007.

[65] W. D. Penny, et al., "Comparing dynamic causal models," Neuroimage, Vol. 22, No. 3, pp. 1157-1172, 2004.

[66] W. D. Penny, et al., "Modelling functional integration: A comparison of structural equation and dynamic causal models," Neuroimage, Vol. 23, Supplement 1, pp. S264S274, 2004.

[67] K. J. Friston, L. Harrison, and W. Penny, "Dynamic causal modeling," Neuroimage, Vol. 19, No. 4, pp. 1273-1302, 2003.

[68] O. David and K. J. Friston, "A neural mass model for MEG/EEG: Coupling and neuronal dynamics," Neuroimage, Vol. 20, No. 3, pp. 1743-1755, 2003.

[69] O. David, D. Cosmelli, and K. J. Friston, "Evaluation of different measures of functional connectivity using a neural mass model," Neuroimage, Vol. 21, No. 2, pp. 659-673, 2004.

[70] B. Horwitz, "Relating fMRI and PET signals to neural activity by means of large-scale neural models," Neuroinformatics, Vol. 2, No. 2, pp. 251-266, 2004.

[71] F. T. Husain, et al., "Relating neuronal dynamics for auditory object processing to neuroimaging activity: A computational modeling and an fMRI study," Neuroi- mage, Vol. 21, No. 4, pp. 1701-1720, 2004.

[72] M. A. Tagamets and B. Horwitz, "Integrating electrophysiological and anatomical experimental data to create a large-scale model that simulates a delayed match-to-sample human brain imaging study," Cerebral Cortex, Vol. 8, No. 4, pp. 310-320, 1998.

[73] J. Loehlin, "Latent variable models: An introduction to factor, path, and structural analysis," 4th edition, Lawrence Erlbaum, Mahwah, New Jersey, 1998.

[74] K. G. Jöreskog and D. Sörbom, "LISREL 8.5 user's reference guide," Scientific Software International, Chicago, 2000.

[75] K. Bollen, "With new incremental structural index for general equation models made," Sociological Methods and Research, Vol. 17, pp. 303-316, 1989.

[76] K. Bollen and J. Long, "Testing structural equation models," Sage, Thousand Oaks, California, 1993.

[77] A. McIntosh, "Understanding neural interactions in learning and memory using function neuroimaging," Annals of the New York Academy of Sciences, Vol. 855, pp. 556 $-571,1998$.

[78] A. McIntosh and F. Gonzales-Lima, "Structural modeling of functional neural pathways mapped with 2-deoxyglucose; effects of acoustic startle habituation on the auditory system 7," Brain Research, Vol. 547, pp. 295-302, 1991.

[79] A. McIntosh and F. Gonzales-Lima, "Network interactions among limbic cortices, basal forebrain, and cerebellum differentiate a tone conditioned as a Pavlovian excitor or inhibitor: Fluorodeoxyglucose mapping and covariance structural modeling," Journal of Neurophysiology, Vol. 72, pp. 1717-1733, 1994.

[80] C. Büchel, J. Coull, and K. Friston, "The predictive value of changes in effective connectivity for human learning," Science, Vol. 283, pp. 1538-1541, 1999.

[81] C. Büchel and K. J. Friston, "Modulation of connectivity in visual pathways by attention: Cortical interactions evaluated with structural equation modelling and fMRI," Cerebral Cortex, Vol. 7, No. 8, pp. 768-778, 1997.

[82] E. Bullmore, et al., "How good is good enough in path analysis of fMRI data?" Neuroimage, Vol. 11, No. 4, pp. 289-301, 2000.

[83] P. Fletcher, et al., "Learning-related neuronal responses in prefrontal cortex studied with functional neuroimaging," Cerebral Cortex, Vol. 9, pp. 168-178, 1999.

[84] S. Grafton, et al., "Network analysis of motor system connectivity in Parkinson's disease: Modulation of thalamocortical interactions after pallidotomy," Human Brain Mapping, Vol. 2, pp. 45-55, 1994.

[85] G. Honey, et al., "Effects of verbal working memory load on corticocortical connectivity modeled by path analysis of functional magnetic resonance imaging data," Neuroimage, Vol. 17, pp. 573-582, 2002.

[86] J. Jennings, A. McIntosh, and S. Kapur, "Mapping neural interactivity onto regional activity: An analysis of semantic processing and response mode interactions," Neuroimage, Vol. 7, pp. 244-254, 1998. 
[87] M. Hollander, "Nonparametric statistical methods," Wiley Series in Probability and Statistics: Applied Probability and Statistic, Wiley, New York, 2nd edition, 1999.

[88] R. Kline, "Principles and practice of structural equation modeling (methodology in the social sciences)," The Guilford Press, 2nd edition, 2004.

[89] B. Byrne, "Structural equation modeling with Amos: BASIC concepts, applications, and programming," Deliver, Lawrence Erlbaum Associates, Reprint edition, 2001.

[90] G. Marrelec, et al., "Using partial correlation to enhance structural equation modeling of functional MRI data," Magnetic Resonance Imaging, Vol. 25, No. 8, pp. 1181$1189,2007$.

[91] G. Marrelec, et al., "Large-scale neural model validation of partial correlation analysis for effective connectivity investigation in functional MRI," Human Brain Mapping, 2008.

[92] B. P. Rogers, et al., "Assessing functional connectivity in the human brain by fMRI," Magnetic Resonance Imaging, Vol. 25, No. 10, pp. 1347-1357, 2007.

[93] B. P. Rogers, et al., "Comment on 'Assessing functional connectivity in the human brain by fMRI'," Magnetic Resonance Imaging, Vol. 26, No. 1, pp. 146, 2008.

[94] M. V. Au Duong, et al., "Modulation of effective connectivity inside the working memory network in patients at the earliest stage of multiple sclerosis," Neuroimage, Vol. 24, No. 2, pp. 533-538, 2005.

[95] M. F. Glabus, et al., "Interindividual differences in functional interactions among prefrontal, parietal and parahippocampal regions during working memory," Cerebral Cortex, Vol. 13, No. 12, pp. 1352-1361, 2003.

[96] R. G. Schlosser, G. Wagner, and H. Sauer, "Assessing the working memory network: Studies with functional magnetic resonance imaging and structural equation modeling," Neuroscience, Vol. 139, No. 1, pp. 91-103, 2006.

[97] R. Schlosser, et al., "Altered effective connectivity during working memory performance in schizophrenia: A study with fMRI and structural equation modeling," Neuroimage, Vol. 19, No. 3, pp. 751-763, 2003.

[98] H. Kondo, et al., "Functional roles of the cingulo-frontal network in performance on working memory," Neuroimage, Vol. 21, No. 1, pp. 2-14, 2004.

[99] J. B. Krause, et al., "Imaging and neural modeling in episodic and working memory processes," Neural Networks, Vol. 13, No. 8-9, pp. 847-859, 2000.

[100] R. A. Charlton, et al., "A structural equation modeling investigation of age-related variance in executive function and DTI measured white matter damage," Neurobiology of Aging, 2007.

[101] J. Rowe, et al., "Attention to action: Specific modulation of corticocortical interactions in humans," Neuroimage, Vol. 17, No. 2, pp. 988-998, 2002.

[102] F. M. Mottaghy, et al., "Systems level modeling of a neuronal network subserving intrinsic alertness," Neuroimage, Vol. 29, No. 1, pp. 225-233, 2006.
[103] K. I. Erickson, et al., "A structural equation modeling analysis of attentional control: An event-related fMRI study," Cognitive Brain Research, Vol. 22, No. 3, pp. 349-357, March 2005.

[104] M. N. Rajah, A. R. McIntosh, and C. L. Grady, "Frontotemporal interactions in face encoding and recognition," Cognitive Brain Research, Vol. 8, No. 3, pp. 259-269, 1999.

[105] G. de Marco, et al., "Changes in effective connectivity during incidental and intentional perception of fearful faces," Neuroimage, Vol. 30, No. 3, pp. 1030-1037, 2006.

[106] J. L. Stein, et al., "A validated network of effective amygdala connectivity," Neuroimage, Vol. 36, No. 3, pp. 736-745, 2007.

[107] B. P. Rogers, J. D. Carew, and M. E. Meyerand, "Hemispheric asymmetry in supplementary motor area connectivity during unilateral finger movements," Neuroimage, Vol. 22, No. 2, pp. 855-859, 2004.

[108] S. T. Grafton, et al., "Network analysis of motor system connectivity in Parkinson's disease: Modulation of thalamocortical interactions after pallidotomy," Human Brain Mapping, Vol. 2, pp. 45-55, 1994.

[109] I. Toni, et al., "Changes of cortico-striatal effective connectivity during visuomotor learning," Cerebral Cortex, Vol. 12, No.10, pp. 1040-1047, 2002.

[110] T. Taniwaki, et al., "Functional network of the basal ganglia and cerebellar motor loops in vivo: Different activation patterns between self-initiated and externally triggered movements," Neuroimage, Vol. 31, No. 2, pp. 745-753, 2006.

[111] J. Zhuang, et al., "Connectivity exploration with structural equation modeling: An fMRI study of bimanual motor coordination," Neuroimage, Vol. 25, No. 2, pp. 462-470, 2004.

[112] A. R. Laird, et al., "Modeling motor connectivity using TMS/PET and structural equation modeling," Neuroimage, 2008.

[113] V. Quaglino, et al., "Differences in effective connectivity between dyslexic children and normal readers during a pseudoword reading task: An fMRI study," Clinical Neurophysiology, Vol. 38, No. 2, pp. 73-82, 2008.

[114] C. H. Fu, et al., "Modulation of effective connectivity by cognitive demand in phonological verbal fluency," Neuroimage, Vol. 30, No. 1, pp. 266-271, 2006.

[115] B. Horwitz, K. J. Friston, and J. G. Taylor, "Neural modeling and functional brain imaging: An overview," Neural Networks, Vol. 13, No. 8-9, pp. 829-846, 2000.

[116] L. Lee, L. Harrison, and A. Mechelli, "A report of the functional connectivity," Workshop, Dusseldorf, pp. 457-465, 2003.

[117] A. McIntosh, "Towards a network theory of cognition," Neural Networks, Vol. 13, pp. 861-876, 2001.

[118] C. Buchel and K. J. Friston, "Modulation of connectivity in visual pathways by attention: Cortical interactions evaluated with structural equation modelling and fMRI," Cerebral Cortex, Vol. 7, No. 8, pp. 768-778, 1997. 
[119] B. Horwitz, "The elusive concept of brain connectivity," Neuroimage, Vol. 19, No. 2 Pt 1, pp. 466-470, 2003.

[120]F. Gonzalez-Lima and A. McIntosh, "Analysis of neural network interactions related to associative learning using structural equation modeling," Mathematics and Computers in Simulation, Vol. 40, No. 1-2, pp. 115-140, 1995.

[121] A. Roebroeck, E. Formisano, and R. Goebel, "Mapping directed influence over the brain using Granger causality and fMRI," Neuroimage, Vol. 25, No. 1, pp. 230-242, 2005.

[122] I. Korhonen, et al., "Linear multivariate models for physiological signal analysis: Theory," Computer Methods Programs in Biomedicine, Vol. 51, No. 1-2, pp. 85-94, 1996.

[123] I. Korhonen, R. Takalo, and V. Turjanmaa, "Multivariate autoregressive model with immediate transfer paths for assessment of interactions between cardiopulmonary variability signals," Medical \& Biology Engineering \& Computer, Vol. 34, No. 3, pp. 199-206, 1996.

[124] K. E. Stephan, et al., "Dynamic causal models of neural system dynamics: Current state and future extensions," Journal of Bioscience, Vol. 32, No. 1, pp. 129-144, 2007.

[125] R. B. Buxton, E. C. Wong, and L. R. Frank, "Dynamics of blood flow and oxygenation changes during brain activation: The balloon model," Magnetic Resonance in Medicine, Vol. 39, No. 6, pp. 855-864, 1998.

[126] K. J. Friston, et al., "Nonlinear responses in fMRI: The Balloon model, Volterra kernels, and other hemodynamics," Neuroimage, Vol. 12, No. 4, pp. 466-477, 2000.

[127] P. J. Basser and D. K. Jones, "Diffusion-tensor MRI: Theory, experimental design and data analysis - a tech- nical review," NMR Biomedicine, Vol. 15, No. 7-8, pp. 456-467, 2002.

[128] P. J. Basser, J. Mattiello, and D. LeBihan, "Estimation of the effective self-diffusion tensor from the NMR spin echo," Journal of Magnetic Resonance B, Vol. 103, No. 3, pp. 247-254, 1994.

[129]D. J. Werring, et al., "The structural and functional mechanisms of motor recovery: Complementary use of diffusion tensor and functional magnetic resonance imaging in a traumatic injury of the internal capsule," Journal of Neurology, Neurosurgery \& Psychiatry, Vol. 65, pp. 863-869, 1998.

[130] D. J. Werring, et al., "A direct demonstration of both structure and function in the visual system: Combining diffusion tensor imaging with functional magnetic resonance imaging," Neuroimage, Vol. 9, pp. 352-361, 1999.

[131] U. C. Wieshmann, et al., "Combined functional magnetic resonance imaging and diffusion tensor imaging demonstrate widespread modified organization in malformation of cortical development," Journal of Neurology, Neurosurgery \& Psychiatry, Vol. 70, pp. 521-523, 2001.

[132] P. J. Olesen, et al., "Combined analysis of DTI and fMRI data reveals a joint maturation of white and grey matter in a fronto-parietal network," Cognitive Brain Research, Vol. 18, No. 1, pp. 48-57, 2003.

[133] F. Gonzalez-Lima and A. R. McIntosh, “Analysis of neural interactions related to associative learning using structural equation modeling," Mathematics and Computers in Simulation, Vol. 40, pp. 115-140, 1995.

[134] A. Mechelli, et al., "Effective connectivity and intersubject variability: Using a multisubject network to test differences and commonalities," Neuroimage, Vol. 17, No. 3, pp. 1459-1469, 2002. 